\title{
Brain Metastases - Clinical Challenges and Recent Advances
}

\author{
Riccardo Soffietti \\ Department of Neuro-Oncology, University of Turin and City of Health and Science University Hospital, Torino, Italy
}

DOI: https://doi.org/10.17925/ENR.2019.14.1.20

$\mathrm{T}$ he treatment of patients with brain metastases (BM) is an important unmet need as, until recently, patients with BM were excluded from clinical studies of systemic therapies. The emergence of molecular targeted therapies has allowed a new treatment approach in patients with targetable mutations, and many of these agents have shown promising intracranial efficacy in lung cancer, breast cancer and melanoma. This editorial summarises the most important new developments and considers the remaining challenges in the treatment of BM.

\section{Keywords}

Brain metastases, targeted therapies

Disclosure: Riccardo Soffietti has nothing to declare in relation to this article.

Review Process: Double-blind peer review.

Acknowledgements: Medical writing assistance, including the preparation of the draft under the guidance of the author, was provided by Katrina Mountfort, Freelance Writer for Touch Medical Media and was supported by Touch Medical Media.

Authorship: The named author meets the International Committee of Medical Journal Editors (ICMJE) criteria for authorship of this manuscript, takes responsibility for the integrity of the work as a whole, and has given final approval for the version to be published.

Received: 23 May 2019

Accepted: 10 July 2019

Citation: European Neurological Review. 2019;14(1):20-1

Corresponding Author: Riccardo Soffietti,

Department of Neuro-Oncology, University

of Turin and City of Health and Science

University Hospital, Via Cherasco 15, 10126

Torino, Italy. E: riccardo.soffietti@unito.it

Support: No funding was received in

the publication of this article.
Brain metastases (BM) are the most frequent among intracranial brain tumours and their incidence is rising as therapeutic advances are improving the survival of patients with advanced cancer. The incidence of BM has been calculated as 9-17\% based on various studies, although the true incidence is thought to be higher. ${ }^{1}$ Patients with BM have a poor prognosis and present a particular therapeutic challenge, as both the intracranial and extracranial disease need to be treated. ${ }^{2}$ The three most common cancers associated with BM are lung cancer, breast cancer and melanoma. Until recently, treatment options for BM have been limited and usually involve multimodality approaches including whole-brain radiation therapy, stereotactic radiation, and surgery. ${ }^{2}$ The use of systemic therapy has been limited in the treatment of BM because large and/or hydrophilic molecules cannot pass the blood-brain barrier (BBB). Patients with BM have generally been excluded from clinical trials of systemic therapy because of their poor prognosis, risk of central nervous system (CNS) haemorrhage, and inefficient drug delivery. ${ }^{3}$ As a result, few clinical trials have specifically addressed this heterogeneous patient population, representing a high unmet medical need.

Enhanced understanding of the molecular processes underlying tumour growth and metastasis has facilitated the development of molecular targeted therapies that are able to cross the BBB. Around $25-40 \%$ of patients with metastatic non-small cell lung cancer (NSCLC) will develop a symptomatic BM. ${ }^{4}$ Prior to the development of molecular targeted therapies, the prognosis for these patients was poor, with a median survival of 3-6 months. ${ }^{5}$ In the past two decades, the discovery of two molecular subgroups of NSCLC - EGFR mutated and ALK rearranged - has transformed outcomes. The first and second generation of ALK inhibitors showed strong efficacy in patients with NSCLC with BM, but resistance to these drugs was a common occurrence. ${ }^{6}$ Next-generation ALK inhibitors, such as brigatinib and lorlatinib were designed to inhibit resistant ALK mutants and have greater penetration of the BBB, and have shown impressive intracranial activity, with overall survival rates exceeding 2 years.? In patients with EGFR mutations and BM, osimertinib has shown improved disease control and longer overall survival. ${ }^{8}$

Breast cancer is the second leading cause of BM; the incidence varies according to the molecular subtype. People with triple negative breast cancer typically have BM early in the disease course and, to date, no effective therapy is available. ${ }^{9}$ Approximately $30-55 \%$ of patients with human epidermal growth factor receptor 2 (HER2)-positive metastatic breast cancer develop BM, but this occurs later in the disease course. ${ }^{10}$ Monoclonal antibodies directed against HER2, such as trastuzumab, have poor penetration of the BBB; however, targeted therapies such as lapatinib, which targets HER2 and EGFR, and neratinib, both in association with capecitabine have shown reduction of the size of CNS lesions in patients with BM. ${ }^{8}$ However, to date, effective treatment options for BM in breast cancer are limited, and currently available drugs used lack specificity for intracerebral tumours.

The risk of BM in melanoma is very high:"11 therapies targeted against the BRAF mutation, specifically vemurafenib and dabrafenib alone or in combination with MEK inhibitors, have shown intracranial activity and improved overall survival in patients with metastatic melanoma. ${ }^{8}$ 
Another major advance in the treatment of advanced solid tumours has been the use of immune checkpoint inhibitors targeting programmed death receptor-1 (PD-1) and programmed death receptor ligand-1 (PD-L1). These include pembrolizumab, nivolumab or atezolizumab, and have shown spectacular efficacy with durable responses in a number of solid tumours (melanoma and NSCLC in particular). To date, only small trials have investigated the intracranial response of patients with BM but early data shows a promising intracranial response rate without neurological side effects. ${ }^{8}$

Adding active systemic therapy to local (radiation, surgery) therapy, which causes temporary disruption of the BBB, is a potentially effective way to improve the outcome of patients with BM. A recent study suggests that the combination of stereotactic radiotherapy with concurrent lapatinib treatment is an effective strategy in metastatic breast cancer. ${ }^{12}$ The combination of BRAF inhibitors with stereotactic radiotherapy is being investigated in the treatment of melanoma. ${ }^{8}$ The immune stimulatory effects of radiation therapy in combination with immunotherapy also appears to be a promising approach. ${ }^{13}$

Despite these advances, significant challenges remain to be addressed in the treatment of BM. Decisions for individualised therapies in patients with BM are often made from biopsies of primary tumours; however, a study found that around $50 \%$ of the actionable mutations in BM were not present in the primary tumour. This study also found that BM can harbour mutations conferring specific drug resistance or activation of alternative signalling pathways interfering with drug activity. ${ }^{14}$ Better understanding of genetic heterogeneity between primary and secondary tumours will lead to improved treatment options.

As newer targeted therapies and immune checkpoint inhibitors are developed, the number and range of potential neurotoxicities also increases. The targeted therapies show excellent penetration of the CNS without significant side effects in the CNS or peripheral nervous system. ${ }^{8}$ Immune checkpoint inhibitors are associated with more serious CNS toxicities; serious neurological adverse events affect approximately $1 \%$ of patients receiving immune checkpoint inhibitors. These are generally transient and include sensory and motor peripheral neuropathies, but rare cases of autonomic neuropathy, Guillian-Barré syndrome, and myasthenia gravis-type syndrome have been reported. Early diagnosis and intervention is essential in these cases. ${ }^{15}$

Clinical research into the treatment of $\mathrm{BM}$ is challenging since patients are often heavily pretreated, and anticancer responses are difficult to observe. Clinical studies often fail to consider the number of $\mathrm{BM}$, the extracranial disease status, prior therapies received or sensitivity to these therapeutic approaches. ${ }^{3}$ The choice of endpoints is also an important consideration when designing a clinical study on BM. In addition to the genetic heterogeneity mentioned above, the uptake and measurement of drug activity on the molecular target are important, as they can vary substantially. The Response Assessment in Neuro-Oncology (RANO) International Group has proposed criteria that should lead to more uniformity in the assessment of BM in clinical trials. ${ }^{16}$

In summary, targeted therapies have improved the survival of patients with NSCLC, breast cancer, and melanoma with actionable mutations, and are likely to play a significant role in the treatment of BM. Improved understanding of the molecular biology of BM should lead to the development of novel immunotherapies and targeted therapies with tumour-specific activity and improved bioavailability beyond the BBB, as well as facilitating advances in radiotherapy and minimally invasive surgery. These advances should lead to better disease control and prolonged survival for patients with BM. $\square$
1. Nayak $L$, Lee $E Q$, Wen PY. Epidemiology of brain metastases. Curr Oncol Rep . 2012:14:48-54.

Soffietti R, Abacioglu U, Baumert B et al Diagnosis and treatment of brain metastases from solid tumors: guidelines treatment of bram metastases from solid tumors: guidelines from the European Association of

3. Lin NU, Lee EQ, Aoyama $H$, et al. Challenges relating to solid tumour brain metastases in clinical trials, part 1: patient population, response, and progression. A report from the RANO group. Lancet Oncol. 2013;14:e396-406.

4. Sorensen JB, Hansen HH, Hansen M, et al. Brain metastases in adenocarcinoma of the lung: frequency, risk groups, and prognosis. J Clin Oncol. 1988;6:1474-80.

5. Patchell RA, Tibbs PA, Walsh JW, et al. A randomized trial of surgery in the treatment of single metastases to the brain $N$ Eng/ J Med. 1990;322:494-500.

6. Toyokawa G, Seto T, Takenoyama M, et al. Insights into brain metastasis in patients with ALK+ lung cancer: is the brain truly a sanctuary? Cancer Metastasis Rev. 2015;34:797-805

7. Petrelli F Lazzari C, Ardito R et al. Efficacy of AlK inhibitors on NSCLC brain metastases: a systematic review and pooled analysis of 21 studies. PLOS One. 2018;13:e0201425.

8. Venur VA, Ahluwalia MS. Targeted therapy in brain metastases: ready for primetime? Am Soc Clin Oncol Educ Book 2016:35:e123-30.

9. Martin AM, Cagney DN, Catalano PJ, et al. Brain metastases in newly diagnosed breast cancer: a population-based study. JAMA Oncol. 2017;3:1069-77.

10. Olson EM, Abdel-Rasoul M, Maly J, et al. Incidence and risk of central nervous system metastases as site of first recurrence in patients with HER2-positive breast cancer treated with adjuvant trastuzumab. Ann Oncol. 2013;24:1526-33.

11. Carlino MS, Haydu LE, Kakavand $H$, et al. Correlation of BRAF and NRAS mutation status with outcome, site of distant metastasis and response to chemotherapy in metastatic melanoma. Br J Cancer. 2014:111:292-9.
12. Parsai S, Miller JA, Juloori A, et al. Stereotactic radiosurgery with concurrent lapatinib is associated with improved local control for HER2-positive breast cancer brain metastases. I Neurosurg. 2019;1-9.

13. Sharabi AB, Tran PT, Lim M, et al. Stereotactic radiation therapy combined with immunotherapy: augmenting the role of radiation in local and systemic treatment. Oncology (Williston Park). 2015;29:331-40.

14. Brastianos PK, Carter SL, Santagata S, et al. Genomic characterization of brain metastases reveals branched evolution and potential therapeutic targets. Cancer Discov. 2015;5:1164-77

15. Blackman JT, Viator TM, Conry RM, et al. Central nervous system toxicities of anti-cancer immune checkpoint blockade. I Neurol Neuromedicine. 2016;1:39-45.

16. Soffietti R, Chiavazza C, Ruda R. Imaging and clinical end points in brain metastases trials. CNS Oncol. 2017;6:243-6. 